\title{
Authentic Dialogue: The Communication of Collaborative Leadership
}

\author{
Jorja Wright \\ School of Business and Leadership \\ University of Charleston-West Virginia
}

\begin{abstract}
Our society has become very complexed and the amount of wicked problems has also increased. Diverse and creative thinking, accompanied by affective leadership, are necessary to reduce the symptoms of healthcare inequities, educational disparities, and climate change. Collaboration is a synergistic process that aims to benefit the group exponentially once all stakeholders work together effectively to reach mutual goals. Authentic dialogue is defined as open and honest conversations that abide by the Habermas speech conditions in which fair exchanges of thoughts lead to detecting and correcting errors (or reconciling differences) amongst engaged actors. This paper: (a) defines authentic dialogue and the conditions for this concept to be used effectively; (b) explains collaborative leadership through the diversity, interdependence, authentic dialogue (DIAD) theory, (c) articulates why understanding systems theory will aid leaders in developing better communication skills; (d) concludes with possible limitations and further studies needed about authentic dialogue within the collaborative process.
\end{abstract}

Key words: Collaborative leadership, authentic dialogue, complexity, systems theory

\section{INTRODUCTION}

Our society has increased in its complexity, which has subsequently led to a plethora of wicked problems. Systems such as healthcare, education, climate control, and government regulation all warrant different perspectives from stakeholders on whether we should do more or less in order to regulate these problems. One thing that citizens can agree upon is all of these systems are certainly not perfect; yet, how to improve these systems remains to be unclear. Diverse and creative thinking, accompanied by affective leadership, are necessary to reduce the symptoms of healthcare inequities, educational disparities, and climate change. Gross [1], McCovery and Matusitz [2], Jäppinen [3] all explain the importance of collaborative processes in order to understand the many wicked problems occuring today. In fact, collaborative efforts are born from necessity, it "emerges from among diverse actors" who share the "mutual desire to achieve goals beyond what any single actor is capable of achieving alone" [1, p. 1]. Collaboration can be viewed from an ethical perspective in an altruisitic nature or from a logical pragmatic view as a necessary process to combat problems larger than one individual [1]. From a complexity or systems perspective, collaboration opens a closed system to stimulation and interactions from outside sources. A case is seen in the Community Partnerships with Health Professions Education (CP/HPE) project which is increasing collaboration amongst actors from the community, academia, and other medical professionals [2]. Policy makers are attempting to open the system of health care for various medical students from purely an academic context (confined "to the classroom or hospital setting") to "actual communities" [2, p. 458]. The collaborative efforts of the CP/HPE [2] example can be categorized as a collaborative process [1]. In fact, Gross identified three disctinct categories of collaboration: process, content, and authority [1]. 
Collaborative processes include the "environments" in which all relevant actors "engage each other" through dialogue, information identification, or trust [1]. Collaborative content are the type of social and/or wicked problems that were mentioned earlier (e.g. healthcare, education, climate control); finally, authority includes collaborative efforts, results, and why these actions are deemed valid in solving the wicked problem [1]. For this paper, collaboration is seen as a dynamic process in which the relevant stakeholders are diverse individuals (including leaders) who share common goals in order to solve an issue [3]; and the mutual solution is greater than the individual solutions of the actors [2]. Collaboration is a synergistic process that aims to benefit the group exponentially once all stakeholders work together effectively to reach this goal. This analysis aims to discuss authentic dialogue within the collaborative process; how it improves communication amongst leaders and other professionals by closing the gap of tribalism [4] within our society; and fostering true innovation to combat the wickedness in many of our social problems. Again, many of these issues may never be solved completely, but the negative consequences can be significantly reduced through effective dialogue and collaboration. This paper: (a) defines authentic dialogue and the conditions for this concept to be used effectively; (b) explains collaborative leadership through the diversity, interdependence, authentic dialogue (DIAD) theory; (c) articulates why understanding systems theory will aid leaders in developing better communication skills; and (d) concludes with possible limitations and further studies needed to better understand authentic dialogue within the collaborative process.

\section{AUTHENTIC DIALOGUE}

Authentic dialogue is based on the great philosophical works of Jürgen Habermas, and his perspective on communicative rationality and John Dewey's pragmatic philosophy [5]. Habermas, Dewey, Innes and Booher's works led to the development of the diversity, interpendence, authentic dialogue (DIAD) theory [5]. According to, Mazutis and Slawinski, dialogue is a vital tool in organizational learning because conversation is the mechanism people use to exchange thoughts and "develop shared understandings" [6, p. 662]. Exchanging ideas and increasing shared understanding amongst actors is tantamount in the collaborative processes because collaboration itself can be considered a social interaction [7]. Hence, authentic dialogue is a valuable tool from a social/relational leadership perspective because it allows leaders and followers to exchange ideas and create shared understandings, in order to enhance learning indvidually and organizationally (Raelin, 2012; Mazutis \& Slawinski, 2007; Raelin, 2014; Hollander, 2011). For this paper, authentic dialogue is defined as open and honest conversations that abide by the Habermas speech conditions in which fair exchanges of thoughts lead to detecting and correcting errors (or reconciling differences) amongst engaged actors (Hollander, 2011; Raelin, 2014; Mazutis \& Slawinski, 2007). It is important for leaders to note that in order for authentic dialogue to be effective, all actors or stakeholders must be committed to the practice in order to move to the next phase of the collaborative process [7]. Leaders that embody authentic leadership theory are able to utilize authentic dialogue effectively because they embody the four main components of authentic leadership: selfawareness, balanced processing, self-regulation, and relational transparency [6]. Authentic leadership (as any other form of leadership) is a complex form of leadership that transcends dimensions and organizational levels, and at the executive level, establishes and cultivates a culture in which authentic dialogue can thrive [6].

\section{Conditions of Authentic Dialogue}

Leaders must realize that their role in the collaborative process (especially amongst followers) is that of facilitator [8]. Leaders heavily influence organizations' cultures through a top-down 
methodology; establishing the right conditions for authentic dialogue to thrive increases the chances of this practice being succesful [8]. Conditions that allow authentic dialogue to flourish include an environment that allows "free exchange so that critical engagement can be sustained" [8]; in other words creating a "democratic order" of leaders that are "meaning makers" who actively engage and participate in community issues to mitigate against wicked problems [7].

Again, this paper stresses the quality of dialogue that is significant to the collaborative process. Dialogue that encourages diverse thinking, sharing, and understanding in a face-to-face format allow learning to take place not only at the individual level, but filtering into the organizational level as well [7]. It is important to note that some conditions may seem conducive to authentic dialogue, however Grandy and Holton [9, p. 432] explain the concept of employee "training opportunities" are not the "right conditions for learning." The etymology of the word, "trainer" in French translates "to drag" in English [9, p. 432]. This explains how new employees are introduced to an organization; they are 'dragged' through a pre-planned one-dimensional knowledge transmission process from expert to novice [9]. However, the aim of effective authentic dialogue is that ideas can be exchanged freely in order to sustain "critical engagement" [8, p. 823].

This type of dialogue allows stakeholders to question perceived "truths," thereby opening the discussion to "new perceptions" and other diverse ideas that can lead to a paradigm shift when looking at a problem or issue [8, p. 823]. Habermas' model of speech conditions grounds the theory of authentic dialogue by defining the terms of rationality [5]. The four speech conditions established by Habermas are located in Table 1 below. Finally, leaders that provide opportunities for this level of dialogue to emerge also allow all stakeholders to free themselves from "authorship and objective standards" by allowing knowledge to be distributed amongst all actors leading to enlightenment and reflexivity [8, p. 823] similar to learning outcomes prescribed by the DEL intellectual community assignments.

Table 1. Habermasian Model of Rational Communication (Raelin, 2012, p. 822; Hollander, 2011, p. 588).

\begin{tabular}{|c|c|}
\hline \multicolumn{2}{|c|}{ Habermasian Model of Rational Communication } \\
\hline Condition 1 - Sincerity & $\begin{array}{l}\text { - Face-to-face discussions } \\
\text { - } \text { Diverse sample group of } \\
\text { - } \text { participants } \\
\end{array}$ \\
\hline Condition 2 - Comprehensibility & $\begin{array}{l}\text { Speech must be: } \\
\text { - } \quad \text { Comprehensible to all actors } \\
\text { - } \quad \text { Logical with evidence to support }\end{array}$ \\
\hline Condition 3 - Interpretation & $\begin{array}{l}\text { - Power must be exercised equally for } \\
\text { all parties } \\
\text { - Information can be accessed by all }\end{array}$ \\
\hline Condition 4 - Normative acceptance & $\begin{array}{l}\text { - All statements are subject to } \\
\text { scrutiny and skepticism } \\
\text { - Best argument (with evidence) } \\
\text { prevails }\end{array}$ \\
\hline
\end{tabular}

\section{Stakeholders Role in Authentic Dialogue}

Grandy and Holton state "all stakeholders" should be involved in the "collaborative process" [9, p. 432]. For authentic dialogue to be effective, the conversation must be based on a "collective 
position" of inquiry that is free from judgment so that all actors and stakeholders involved feel "competent, trusted and valued" [8, p. 821]. The trust, social and intellectual capital gained from including stakeholders in collaborative conversations are invaluable to cultivating a culture of inquiry, "knowledge creation, and resource acquisition" therfore leading to rich "interorganizational" and "intraorganizational social networks" [8, p. 824]. For example, the case of The University of Colorado Digital Health Consortium Intitiative explains important stakeholder involvement in the collaborative implementation of the Healthcare Information Technology for Economic and Clinical Health (HITECH) Act [10]. HITECH Act involves stakeholders on the delivery side of the health care system, including: students, physicians, nurses, nurse executives, healthcare administrators, and healthcare information technology (IT) professionals (Khuntia et al., 2014; Burgess \& Curry, 2014). Policy changes in health care delivery have called for innovative measures with implementing new health care delivery practices due to the investments of many stakeholders [10]. Brainstorming and pooling existing resources with diverse stakeholders increase the "success and sustainability" of collaborative health care delivery projects [11, p. 533]. Raelin reiterates the importance of relevant stakeholders being present when discussing wicked problems because it fulfills Habermasians conditions for rational communication [8]. Collaborative forums that include all relevant stakeholders uphold Habermas' speech conditions through:

a) Sincerity - allowing everyone to listen together in a face-to-face fashion in order to see the speaker's sincerity;

b) Comprehensibility - understanding the speaker and respecting the "coherence" of the others' views;

c) Interpretation - suspending preconceived notions so that all forum attendees can ask questions without fear of judgment from other participants;

d) Normative acceptance - acknowledging all participants' positions of power so that no actor can manipulate the power of another and allowing all actors to freely agree (or disagree) with various points raised during collaborative forum [8].

As in the University of Colorado case, authentic dialogue that occurs in collaborative forums (as mentioned above) are vital to the effectiveness of collaborative efforts when faced with wicked problems. Health care organizations must identify and utilize all stakeholders in order to "leverage the skills/knowledge base" within these social networks and to close the gaps in knowledge amongst academics, politicians, health care professionals and the rest of the community in order to improve the health care system [10, p. 34]. Next, I will discuss the DIAD theory of collaborative rationality and its significance to the authentic dialogue concept.

\section{DIAD THEORY OF COLLABORATIVE RATIONALITY}

Innes and Booher [4] popularized the concept of collabroative rationality which is a blend of Habermas's theory of communicative rationality and Dewey's pragmatic philosophies [5]. Diversity, interdependence, authentic dialogue theory of collaborative rationality is "both descriptive and normative theory of collaborative processes" [1, p. 18]. Moreover, particpants of collaborative processes based on the DIAD theory are stakeholders that have something to gain from the collaboration as opposed to being subscribers to the ethical perspective of altruism, in other words being self-less [1]. Collaborative processes are considered rational once they meet these seven conditions [12]:

1. Diverse particpants with varying points of view (POV).

2. All actors are engaged and interested in the problem.

3. Each participant has equal opportunity to express their POV, and are delayed from taking sides once all participants are able to explain their individual points.

4. All actors meet face-to-face, and each are encouraged to speak up. 
5. Technical expertise along with community knowledge are both included in the dialogue.

6. All issues are "on the table" in order to increase dialogue or allow participants to reframe the issue generating innovative solutions [12, p. 3].

7. All participants work through issues using creative strategies to "satisfy the significant concerns of each member" [12, p. 3].

As mentioned in Planning with Complexity: the objective of collaborative rationality is for all actors to reach a consensus during the collaborative process [4]. The seven conditions of collaborative rationality are similar to the four main conditions of Habermasian's rational communication model - sincerity, comprehensibility, interpretation, normative acceptance [8]. However, collaborative rationality elaborates on Habermasian's model in that it speaks on the collaborative process beyond conversation, including diversity of relevant actors, stakeholders and considers a "full range of views" [12, p. 4]. Authentic dialogue can be seen as the catalyst that ignites a collaborative process; as in chemistsry, in which a catalyst expedites a chemical reaction without being consumed by the process. While discussions tend to be linked to a specific position or "adversarial" in nature, authentic dialogue is open to multiple perspectives, free of judgment, and links the other aspects of collaborative rationality together [13, p. 184]. Figure 1 illustrates the DIAD theory; DIAD theory is successful once all participants are pursuing their individual interests but still making rational choices [1].

As seen in the figure, authentic dialogue is the bond that connects "participants" with

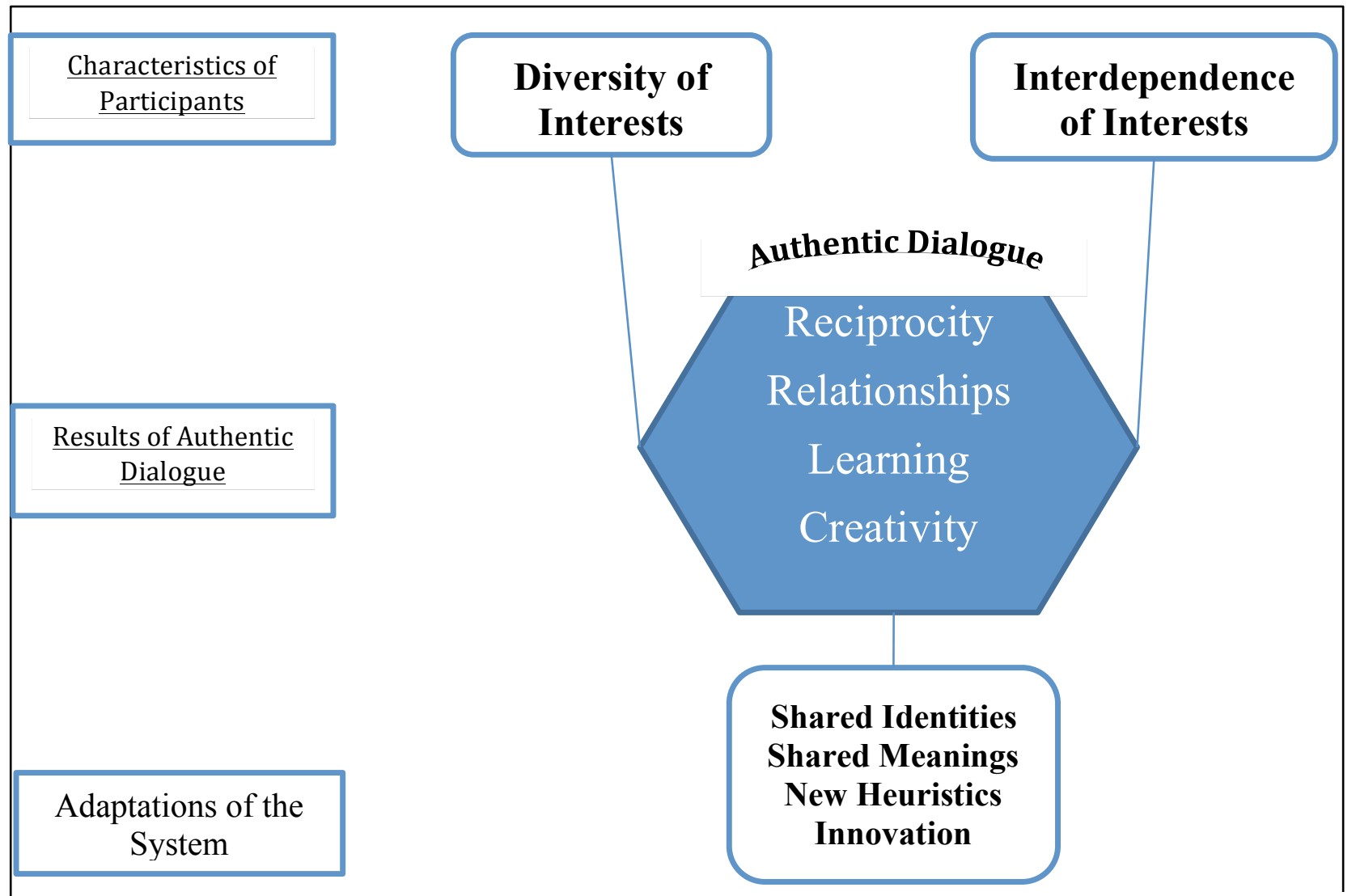

Figure 1. Model of DIAD theory of collaborative rationality [1, p. 33]. Figure 2. Model of DIAD theory of collaborative rationality (Gross, 2012, p. 33).

interdependent interests. The DIAD theory only works with partipants that are mutally interested in benefitting and offering something to others within the collaborative process [1]. In fact, Gross elaborates about research which show "reciprocity and cooperation based on rational choice" are conducive to DIAD theory being successful [1, p. 20]. Participants must reach the conditions of authentic dialogue in order to exploit on the relationship between 
diversity and interdependence [1]. In the end, skilled facilitation is needed in order to guide participants into listening to each other and the other "deliberative norms" needed for authentic dialogue [1, p. 34]. Next, I will discuss the tenets of systems theory and how it correlates with authentic dialogue.

\section{SYSTEMS THEORY AND AUTHENTIC DIALOGUE}

Systems theory explains phenomenons from the perspective of a dynamic process with interrelated units. The tenets of systems theory are as follows: 1) single units are interconnected; and 2) the whole equals more than the sum of individual units [2]. Systems theory has five basic beliefs, which include:

a) Systems contain units, variables, elements that are concrete, abstract, or both.

b) Systems have their own unique qualties or properties.

c) System units are interconnected.

d) Systems occur in particular environments.

e) Two kinds of systems exist: open and closed [2].

Open systems interact with its environment and are adaptable while closed systems do not. Interacting with elements outside systems makes open system more sustainable and have an increased chance of survival [2].

A systems approach to combating wicked problems is beneficial because it allows for diverse stakeholders to contribute to the solution and gain from the discursive exchange [14]. Further, a systems theory approach to collaboration allows participants to cultivate a creative, innovative environment which leads to new paradigms of collecting, utilizing, and allocating resources more effectively [14]. Creative ways of utilizing limited resources is even more significant due to the financial constraints many organizations faced and yet increased demands for services [15]. Returning to the health care example of The University of Colorado Digital Health Consortium Intitiative, collaboration was an integral part of the HITECH Act which improves health care delivery through technology implmentation. Demand for vital health care services have steadily increased, however, collaborative efforts are inhibited due to bureacratic nature of some organizational structures. VanVactor calls these bureacratic barriers "inter-organizational barriers and intra-organizational silos" in which each leader has a "set of resources" within his or her control [16, p. 555]. These barriers significantly inhibit collaborative processes because they close the system and reduce its sustainability. Authentic dialogue cannot flourish in a closed system because the main element it needs to be effective is diversity which comes from units (participants or stakeholders) outside of the system. During dialogue, exhanges occur not only from "leader-to-group member," but also from "member-tomember" [17, p. 1025]. In this way, collaborative (group) leadership emerges because "leadership functions are transferred among group members" [17, p. 1025]. Quanity of meetings also play a role in cultivating an environment that is advantageous for authentic dialogue. Shrimali, et al. stress scheduling "regular meetings" amongst relevant stakeholders because these provide both "informal and formal opportunities for learning and dialogue" [18, p. 376]. Thus, communication behaviors within a system are important to successful collaboration processes, because authentic dialogue is the link between participants and interdependent interests.

\section{LIMITATIONS}

Though authentic dialogue comes with many benefits to the collaborative process, there are still a few limitations that halt its implementation. Some practitioners will object to implementing the practice due to costs, time constraints, politics, or claims that it is simply 
impractical [12]. Other limitations include, unable to pay for a trained facilitator, a quick decision is needed, incapable of convincing upper management that process is needed, or cannot bring relevant stakeholders together in order for dialogue to occur [12]. Another challenge with authentic dialogue is encouraging participants to be true and authentic to himor herself when speaking on issues as opposed to providing "only superficial explanations for what they believe in" [8, p. 828]. Researchers reassure that some practitioners have been able to conquer many of these limitations. For example, organizations can train employees to be neutral facilitators of collaborative projects outside of their department so that authentic dialogue can occur [12]. Furthermore, the identification of stakeholders and the perception of 'what is fair' is another limitation that needs deeper study. Raelin refers to these silent actors as "hidden voices" because their "interests, for whatever reason, are not considered" [8, p. 829]. It is vital for authentic dialogue to be operative, so these "hidden voices" must gain their own platform to express themselves. Superiors must not speak on behalf of these voices because it is counterproductive to the practice of authentic dialogue [8]. Finally, Hollander explains that face-to-face human interaction are "susceptible" to "prejudice and intolerance of those who are different" [5, p. 589]. Group think, exaggertion, posturing, or deception are a few of the other issues that occur within dialogical exchanges [5]. Fortunately, leaders can consciously implement strategies so that learning processes occur during authentic dialogue [5].

\section{CONCLUSION}

This paper examines the positive effects of authentic dialogue and its relation to the DIAD theory. Dialogue differs from discussion due to very specific conditions such as diversity of participants, equal distribution of power, and all statements are subject to inquiry. DIAD theory of collaborative rationality is an ideal; in reality, all collaborative exchanges are wrought with complications and limitations. Every organization faces challenges when seeking partnerships, and the lack of research in the details of communicative exchanges within collaborative rationality complicates collaborative efforts even more [19]. One major take away for executive leaders is to understand their own engagement in the collaborative process. In fact, Kumar et al. explains that organiztions that are willing "to establishish meaningful and effective relationships with its partner groups" are more likely to adopt collaborative efforts [19]. Executive administrators must decide if their lack of collaborative partnerships is a result of "inability," which is "lack of empowerment within an organization, or "unwillingness," which is "a bureaucratic mind-set" $[19$, p. 570]. It is important for administrators to raise awareness: first, of the benefits of collaborative partnerships in that it increases an organizations sustainability and helps to mitigate a plethora of issues; second, the benefits of authentic dialogue because this type of dialogue leads to successful collaborative efforts [20]. As the name suggests, collaboration is a joint effort that requires engagement from all parties in order for it to be successful. Judgment free dialogue increases individual and organizational learning, which are all vital to authentic dialogue and collaborative efforts. The many wicked problems in our society require input from various professionals, scholars, researchers, administrators, and other community members.

\section{References}

[1] P. Gross, "A Q methodology analysis of individual perspectives of public decision making inflluences of collaborative processes," UMI Dissertation Publishing , 2012.

[2] J. McCovery and J. Matusitz, "Assessment of collaboration in U.S. health care delivery: a perspective from systems theory," Social Work in Public Health, vol. 29, no. 5, pp. 451-461, 2014. 
[3] A. Jäppinen, "Co-dynamics in engineering innovations through collaborative leadeship - a complexity-based approach," in CO-CREATE 2013 : The Boundary-Crossing Conference on Co-Design in Innovation, Helsinki, 2013.

[4] J. Innes and D. Booher, Planning with complexity: An introduction to collaborative rationality for public policy, Milton Park: Routledge, 2010.

[5] J. Hollander, "Approaching an ideal: using technology to apply collaborative rationality to urban planning processes," Planning Practice and Research, vol. 26, no. 5, pp. 587-596, 2011.

[6] D. Mazutis and N. Slawinski, "The art of conversation: how authentic leaders influence organizational learning," in Proceedings of OLKC 2007 - "Learning Fusion", 2007.

[7] J. Raelin, "Imagine there are no leaders: Reframing leadership as collaborative agency," Leadership, vol. 0, no. 0, pp. 1-28, 2014.

[8] J. Raelin, "The manager as facilitator of dialogue," Organization, vol. 20, no. 6, pp. 818-839, 2012.

[9] G. Grandy and J. Holton, "Leadership development needs assessment in healthcare: a," Leadership \& Organization Development Journal, vol. 34, no. 5, pp. 427-445, 2013.

[10] J. Khuntia, J. Karimi, M. Tanniru and A. Meyers, "The University of Colarado digital health consortium inititative: a collaborative model of education, research and service," Journal of Commmercial Biotechnology, vol. 20, no. 3, pp. 31-37, 2014.

[11] C. Burgess and M. Curry, "Transforming the health care environment collaborative," AORN Journal, vol. 99, no. 4, pp. 529-539, 2014.

[12] J. Innes, "Collaborative rationality for planning practice," The town planning review, vol. 84, no. 1, pp. 1-4, 2016.

[13] M. Fletcher, M. Morrison, D. Giles and J. Kedian, "Leadership development as a dialogic process the rationale and concept of an international leadership institute," International Journal of Leadership in Education, vol. 19, no. 2, pp. 182-202, 2016.

[14] A. Thorkildsen and M. Ekman, "The complexity of becoming collaborative planning and cultural heritage," Journal of Cultural Heritage Management and Sustainable Development, vol. 3, no. 2, pp. 148-162, 2013.

[15] H. Taylor and R. Morse, "Collaborative leadership development for local government officials: exploring competencies and program impact," Public Administration Quarterly, vol. 37, no. 1, pp. 72-103, 2013.

[16] J. VanVactor, "Collaborative leadership model in the management of health care," Journal of Business Research, vol. 65, pp. 555-561, 2012.

[17] M. Kramer and D. Crespy, "Communicating collaborative leadership," The Leadership Quarterly, vol. 22, pp. 1024-1037, 2011.

[18] B. Shrimali, J. Luginbuhl, C. Malin, R. Flournoy and A. Siegel, "The building blocks collaborative: advancing a life course approach to health equity through multi-sector collaboration," Maternal Child Health Journal, vol. 18, pp. 373-379, 2014.

[19] S. Kumar, S. Kant and T. Amburgey, "Public Agencies and Collaborative Management Approaches," vol. 39, no. 5, pp. 569-611, 2007.

[20] D. Garrison and N. Vaughan, "Institutional change and leadership associated with blended learning innovation: Two case studies," Internet and Higher Education, vol. 18, pp. 24-28, 2012. 